\title{
Paediatric supracondylar humeral fractures: a technique for safe medial pin passage with zero incidence of iatrogenic ulnar nerve injury
}

Chin Yee $\underline{W o O}^{1}$, MBBS, MRCSEd, Han Loong $\underline{H o}^{1}$, MBBS, Mohammad Bin Zainuddin Ashik ${ }^{1}$, MMED, FRCSEd, Kevin Boon Leong Lim ${ }^{1}$, FRCS, FRCSEd

\begin{abstract}
INTRODUCTION The cross Kirschner wire (K-wire) configuration in closed reduction and percutaneous pinning of paediatric supracondylar humeral fracture affords superior stability. However, medial pin placement presents a risk of iatrogenic ulnar nerve injury. This study describes, in step-by-step detail, another safe method of percutaneous medial pin insertion.

METHODS The technique involved placing the patient's arm in external rotation, with elbow flexed no more than $45^{\circ}$ after closed reduction. The surgeon held the K-wire close to its sharp end to pass it percutaneously onto the medial epicondyle, then adjusted his grip toward the blunt end. After fluoroscopy check, the wire driver was engaged and an anteriorly directed force was applied to the distal humerus fragment using the thumb of the surgeon's free hand. The K-wire was inserted at a $45^{\circ}$ angle to the longitudinal axis of the humerus shaft. Clinical notes and radiographs of patients who underwent surgery with this technique from 2006 to 2008 were reviewed.

RESULTS A total of 125 patients (84 boys, 41 girls) were included, with a mean age of 7.1 (range 2-14) years. Most injuries were left-sided $(72.8 \%, n=91$, vs. right: $27.2 \%, n=34) .72(57.6 \%)$ patients had two-pin cross K-wire configuration, while $53(42.4 \%)$ patients had an additional lateral pin inserted. No patient had postoperative ulnar neuropathy. There were no complications of non-union, malunion or infection.

CONCLUSION This safe method of medial pin placement for surgical stabilisation of paediatric supracondylar humeral fractures is easily learnt and reproducible, and produces excellent results.
\end{abstract}

Keywords: children, elbow, humerus, k-wiring, percutaneous pinning

\section{INTRODUCTION}

Supracondylar fractures of the humerus are the second most common fractures in children, making up nearly $60 \%$ of all paediatric elbow fractures. ${ }^{(1)}$ Based on the Wilkins-modified Gartland classification, Type IIb and III fractures are unstable and should be treated operatively by closed reduction and percutaneous pinning as the established gold standard. ${ }^{(1)}$ The initial technique was first described in 1948.(2)

Biomechanically, the cross Kirschner wire (K-wire) configuration (Fig. 1; one medial and one lateral, with intersection of both wires just proximal to the fracture site) is the strongest, especially for resisting axial rotation, as tested on synthetic bone and human cadaver models. ${ }^{(3,4)}$ However, medial pin insertion presents a risk of iatrogenic injury to the ulnar nerve due to the anatomical location of the ulnar nerve in the groove just posterior to the medial epicondyle; the incidence of iatrogenic ulnar nerve injury has been reported to be as high as $5 \%{ }^{(5)}$ This led to the development of the lateral-only configuration, which is favoured by some and involves three lateral pins (proven by Zionts et $\mathrm{al}^{(4)}$ to possess almost equal stability as the cross K-wire configuration), two diverging lateral pins by Skaggs et al ${ }^{(6)}$ and two converging lateral pins in Dorgan's technique. ${ }^{(7)}$

Currently, safe medial pin insertion techniques that have been described to minimise iatrogenic ulnar nerve injury include 'milking' the soft tissue posteriorly using the thumb to protect the ulnar nerve and positioning the elbow in extension for medial pin insertion, as mentioned in a textbook ${ }^{(8)}$ and in the study by Edmonds et al. ${ }^{(9)}$ The present study further emphasises the importance of these steps and, additionally, refines the technique with attention to details such as pin holding, positioning and passage.

\section{METHODS}

This was a retrospective study that involved review of the clinical notes (e.g. surgical notes and consultation notes from followup visits) and radiographs of patients who underwent closed reduction and percutaneous pinning for isolated extensiontype supracondylar humeral fractures at the Department of Orthopaedic Surgery, KK Women's and Children's Hospital, Singapore, from 2006 to 2008 using the technique described herein. Evaluation at clinic follow-up visits was done by the attending doctor in the clinic and not by the surgical team. Patients with flexion-type supracondylar humeral fractures, multiple fractures on the same limb and preoperative ulnar nerve neuropathy were excluded from the study.

In the technique used by surgeons in our study, the medial pin was inserted first, before the lateral pin(s). After closed reduction of the fracture, the patient's arm was placed in external rotation 

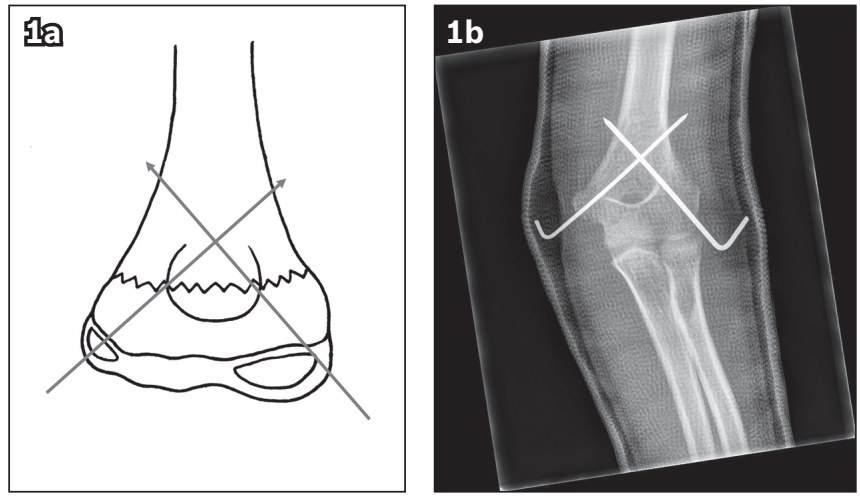

Fig. 1 (a) Illustration and (b) radiograph show the cross Kirschner wire configuration for patients with paediatric supracondylar humeral fractures.

on the image intensifier (detector of the C-arm), so that true lateral radiographs of the distal humerus could be readily obtained. In addition, the elbow was well supported in this position, which facilitated palpation of the medial epicondyle and passage of the medial pin thereafter.

To identify the entry point, the medial epicondyle was identified, and with the tip of the fingernail of the surgeon's non-dominant thumb at the edge of the medial epicondyle, the ulnar nerve was located to be in the vicinity of the pulp of the surgeon's non-dominant thumb. The surgeon held the smooth K-wire close to its sharp end to pass it percutaneously onto the medial epicondyle, with the patient's elbow flexed no more than $45^{\circ}$ (Fig. 2a). The use of the precise pincer grip to hold the wire close to its sharp end with controlled identification of the medial epicondyle reduced the risk of slippage or plunging in of the wire and the need to make multiple attempts or passes. The entry point position was maintained by gently applying pressure to rest the K-wire firmly on the medial epicondyle, as the surgeon's grip was adjusted away to the middle or toward the blunt end of the K-wire (Fig. 2b). The starting point was confirmed with fluoroscopy and the wire driver was engaged onto the K-wire in preparation for drilling (Fig. 2c). The elbow was maintained in $45^{\circ}$ or less of flexion throughout in preparation for K-wire insertion, so as to prevent anterior subluxation of the ulnar nerve over the medial epicondyle (which happens in flexion). For the passage of the medial pin, an anteriorly directed force was applied to the distal humeral fragment using the thumb of the surgeon's free hand (Fig. 2d) to correct for residual extension. The K-wire was inserted at an angle of $45^{\circ}$ to the longitudinal axis of the humerus shaft. The K-wire placement was checked with fluoroscopy in both the lateral and anteroposterior views. Throughout the process, the surgeon and an assistant watched for any twitching of the ipsilateral ring and little fingers, which would have indicated that the wire was in contact with or in close proximity to the ulnar nerve.

\section{RESULTS}

A total of 125 patients (84 boys, 41 girls; mean age 7.1 [range 2-14] years) with paediatric supracondylar humeral fractures were included in the study. Both left-sided ( $\mathrm{n}=91,72.8 \%$ ) and right-sided $(\mathrm{n}=34,27.2 \%)$ injuries were seen. $72(57.6 \%)$ patients had a two-pin cross K-wire construct, while 53 (42.4\%) patients had a second lateral pin inserted (Fig. 3).

All patients attended their required follow-up visits and there were no defaulters. There was no documented injury to the ulnar nerve or other nerves, and no loss of alignment or residual joint stiffness in this series. There were also no complications of nonunion, malunion or infection on follow-up.

\section{DISCUSSION}

While the cross K-wire configuration used for patients with paediatric supracondylar humeral fractures is superior in resisting axial rotation, it requires insertion of a medial pin, and this presents a risk of iatrogenic injury to the ulnar nerve. This resulted in the development of and preference for the lateral-only configuration by some surgeons, with good outcomes. Studies have shown that the lateral-only configuration is comparable to the cross K-wire configuration in terms of outcomes of instability and incidence of nerve injury. ${ }^{(6,7)}$ However, the authors believe that in certain fracture configurations, especially those with medial-sided comminution, the medial pin is still required for a stable construct, as postoperative instability has been described in such patients with lateral-only pinning.

Previously described techniques of medial pinning involve: maintaining flexion of the elbow; ${ }^{(10)}$ using accurately drawn lines on the skin around the elbow; ${ }^{(10)}$ using a small incision over the medial epicondyle for direct visualisation; ${ }^{(11,12)}$ and using a nerve stimulator to identify the location of the ulnar nerve. ${ }^{(13)}$ Our proposed technique compared favourably with these other methods in reducing the risk of ulnar nerve injury, and does not require the use of additional equipment or result in an additional open wound for the patient.

Our technique of using a two- or three-finger grip allowed for precise and controlled identification of the medial epicondyle. If the wire tip was applied on the prominence of the medial epicondyle and held steady while the drill was mounted onto it, only one pass was necessary. Wire slippage or plunging and ulnar nerve injury was rare. We do not recommend mounting the K-wire on the drill and then locating the starting point on the medial epicondyle. When the wire is held far from its leading sharp end, control is imprecise and there is a tendency to make multiple passes, which increases the risk of ulnar nerve injury.

After the starting point is confirmed, Swenson ${ }^{(2)}$ initially proposed drilling the pin on an acutely flexed arm to maintain reduction of the fracture. However, this is not recommended, and we feel that it is critical for the pin to be passed with the elbow in relative extension so as to prevent anterior subluxation of the ulnar nerve over the medial epicondyle. The surgeon can readily correct residual extension simply by applying an anteriorly directed force on the distal humeral fragment using the thumb of the free hand when advancing the K-wire. We believe that iatrogenic ulnar nerve injury almost always results from an incorrectly placed pin (the starting point is often inferior to and/or posterior to the medial epicondyle). Although there have been reports showing that the ulnar nerve may migrate to the anterior aspect of the medial epicondyle in about $20 \%$ of cases in children, ${ }^{(14-16)}$ in our 

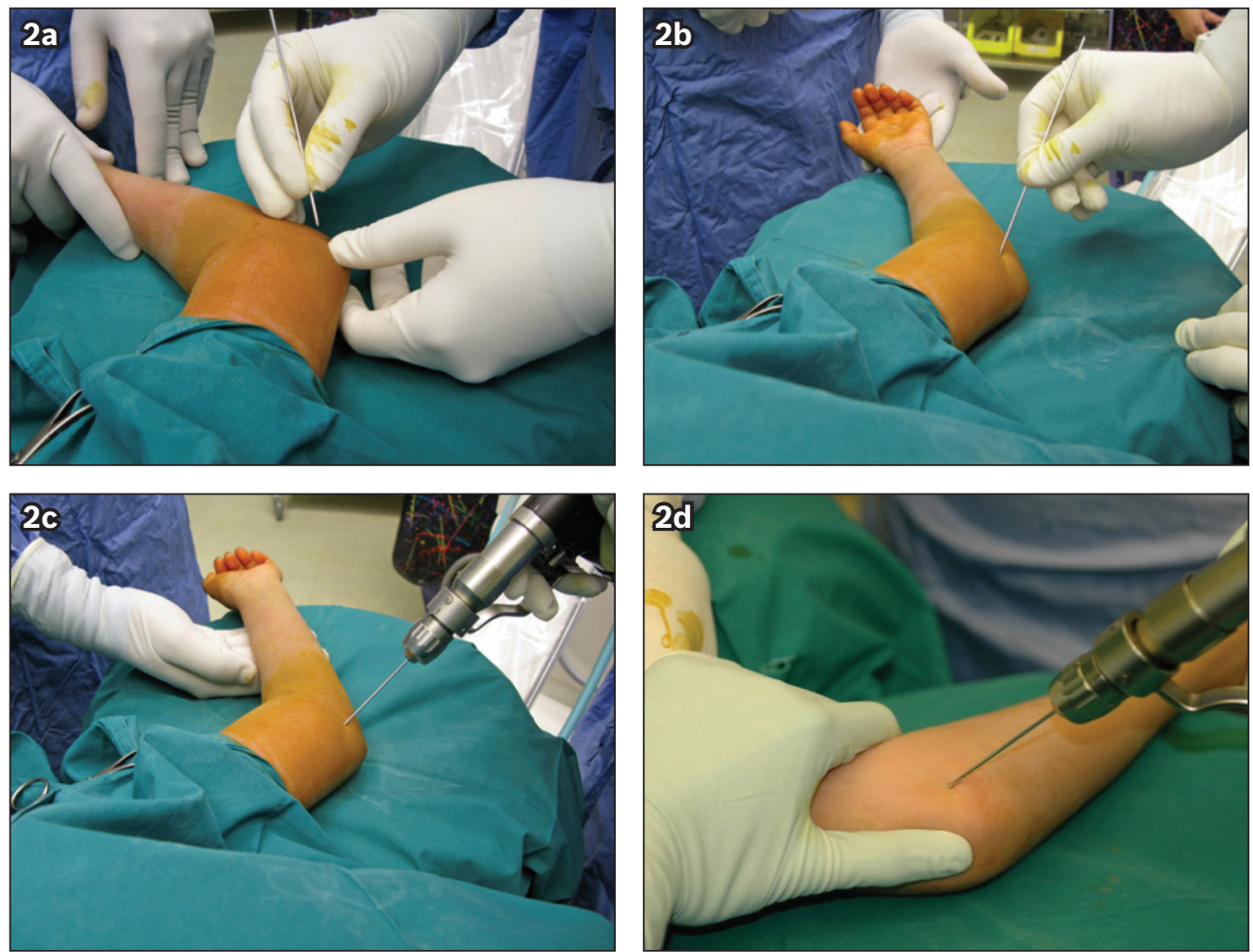

Fig. 2 Photographs show the steps for percutaneous medial pin insertion in patients with paediatric supracondylar humeral fractures. (a) To identify the entry point, the surgeon holds the smooth Kirschner wire (K-wire) close to its sharp end to pass it percutaneously onto the medial epicondyle, with the patient's elbow flexed no more than $45^{\circ}$. (b) Gentle pressure is applied to rest the K-wire firmly on the medial epicondyle, as the surgeon's grip is adjusted to the middle or blunt end of the K-wire. (c) The starting point is confirmed with fluoroscopy and the wire driver is engaged onto the K-wire in preparation for drilling. (d) An anteriorly directed force is applied to the distal humeral fragment using the thumb of the surgeon's free hand to correct for residual extension. The $\mathrm{K}$-wire is inserted at $45^{\circ}$ to the longitudinal axis of the humerus shaft.
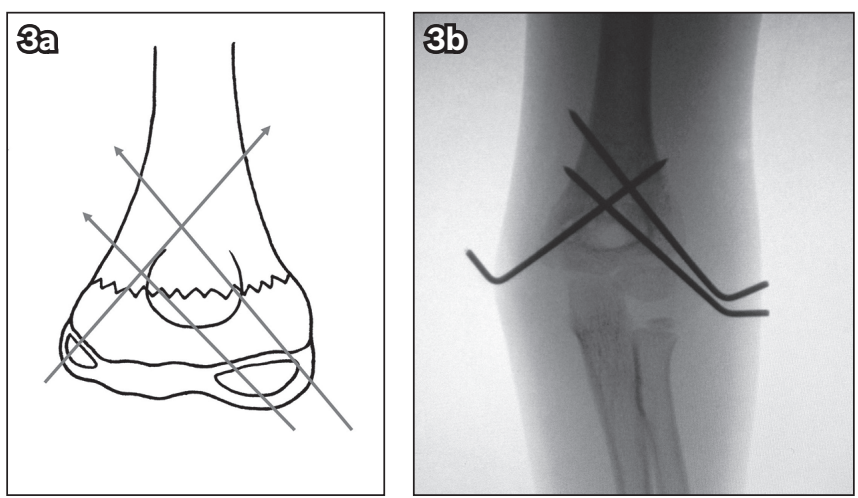

Fig. 3 (a) Illustration and (b) intraoperative radiograph show the cross Kirschner wire configuration using one medial and two lateral pins for patients with paediatric supracondylar humeral fractures.

experience with the present cohort, we did not encounter a single case of twitching of the ring and little fingers when the medial pin was advanced. Another proposal was that by Gordon et al ${ }^{(11)}$ and Green et al, ${ }^{(12)}$ who recommended the use of a small medial incision to directly visualise the nerve. For adequate visualisation, the incision made must be at least $10 \mathrm{~mm}$ in length. This has not proven necessary in our experience. The use of an incision increases the risk of scarring and infection, and results in a surgical scar, which most parents would wish to avoid for their children.

One limitation of this study is that it was a retrospective review of a single surgeon series in which all the procedures were performed by the senior author or by trainees under his direct supervision in the operating theatre, although the follow-up clinic review and assessment was done by the attending doctor at the clinic and not the surgical team. Additionally, the number of attempts at passing the K-wire was not recorded.

In conclusion, this study described a modification and refinement of the technique of inserting the medial pin in a stepby-step fashion, with attention to details such as pin holding, handling and passage. The technique described complements the existing recommendations to minimise iatrogenic ulnar nerve injury during medial pin placement for the stabilisation of paediatric supracondylar fractures of the humerus. It is easily learnt and reproducible, and produces good results.

\section{REFERENCES}

1. Gartland JJ. Management of supracondylar fractures of the humerus in children. Surg Gynecol Obstet 1959; 109:145-54.

2. Swenson AL. The treatment of supracondylar fractures of the humerus by Kirschner-wire transfixion. J Bone Joint Surg Am 1948; 30A:993-7.

3. Lee SS, Mahar AT, Miesen D, Newton PO. Displaced pediatric supracondylar humerus fractures: biomechanical analysis of percutaneous pinning techniques. J Pediatr Orthop 2002; 22:440-3.

4. Zionts LE, McKellop HA, Hathaway R. Torsional strength of pin configurations used to fix supracondylar fractures of the humerus in children. J Bone Joint Surg Am 1994; 76:253-6.

5. Skaggs DL, Hale JM, Bassett J, et al. Operative treatment of supracondylar fractures of the humerus in children. The consequences of pin placement. J Bone Joint Surg Am 2001; 83-A:735-40.

6. Skaggs DL, Cluck MW, Mostofi A, Flynn JM, Kay RM. Lateral-entry pin fixation in the management of supracondylar fractures in children. J Bone Joint Surg Am 2004; 86-A:702-7.

7. Shannon FJ, Mohan P, Chacko J, D'Souza LG. "Dorgan's" percutaneous lateral cross-wiring of supracondylar fractures of the humerus in children. J Pediatr Orthop 2004; 24:376-9.

8. Herring JA. Upper Extremity Injuries, Fractures About the Elbow. In: Tachdjian's Pediatric Orthopaedics Third Edition. Philadelphia: Saunders, 2002: 2153-55. 
9. Edmonds EW, Roocroft JH, Mubarak SJ. Treatment of displaced pediatric supracondylar humerus fracture patterns requiring medial fixation: a reliable and safer cross-pinning technique. J Pediatr Orthop 2012; 32:346-51.

10. Reynolds RA, Mirzayan R. A technique to determine proper pin placement of crossed pins in supracondylar fractures of the elbow. J Pediatr Orthop 2000; 20:485-9.

11. Gordon JE, Patton CM, Luhmann SJ, Bassett GS, Schoenecker PL. Fracture stability after pinning of displaced supracondylar distal humerus fractures in children. J Pediatr Orthop. 2001; 21:313-8.

12. Green DW, Widmann RF, Frank JS, Gardner MJ. Low incidence of ulnar nerve injury with crossed pin placement for pediatric supracondylar humerus fractures using a mini-open technique. J Orthop Trauma 2005; 19:158-63.

13. Michael SP, Stanislas MJ. Localization of the ulnar nerve during percutaneous wiring of supracondylar fractures in children. Injury 1996; 27:301-2.

14. Childress HM. Recurrent ulnar-nerve dislocation at the elbow. Clin Orthop Relat Res 1975; (108):168-73.

15. Zaltz I, Waters PM, Kasser JR. Ulnar nerve instability in children. J Pediatr Orthop 1996; 16:567-9.

16. Okamoto M, Abe M, Shirai H, Ueda N. Morphology and dynamics of the ulnar nerve in the cubital tunnel. Observation by ultrasonography. J Hand Surg $\mathrm{Br}$ 2000; 25:85-9. 